IP/BBSR/95-51

hep-th/9506037

\title{
Symmetries of Heterotic String Theory
}

\author{
Anindya K. Biswas ${ }^{1}$, Alok Kumar ${ }^{1,2}$ and Koushik Ray ${ }^{1}$ \\ 1. Institute of Physics, \\ Bhubaneswar 751 005, INDIA \\ email: akb, kumar, koushik@iopb.ernet.in \\ 2. ICTP, 34100 Trieste, Italy \\ email: alok@ictp.trieste.it
}

October 7, 2018

\begin{abstract}
We study the symmetries of the two dimensional Heterotic string theory by following the approach of Kinnersley et al for the study of stationary axially-symmetric Einstein-Maxwell equations. We identify the finite dimensional symmetries which are the analogues of the groups $G^{\prime}$ and $H^{\prime}$ for the Einstein-Maxwell equations. We also give the constructions for the infinite number of conserved currents and the affine $\hat{o}(8,24)$ symmetry algebra in this formulation. The generalized Ehlers and Harrison transformations are identified and a parallel between the infinite dimensional symmetry algebra for the heterotic string case with $\hat{s l}(3, R)$ that arise in the case of Einstein-Maxwell equations is pointed out.
\end{abstract}

\footnotetext{
${ }^{1}$ Permanent address
} 


\section{Introduction}

There has been a resurgence of interest in the investigatons of duality symmetries [1, 2, [3] in string theory. In a series of interesting papers, Sen gave the explicit realization of S- and T-dualities for the torus compactified heterotic string theories in various dimensions «, 5, 6, 7, 8], exploited them for obtaining the solitonic spectrum of these theories and argued in favour of the nonperturbative validity of the S-duality symmetry. Partly inspired by these results, further studies led to the interesting developments towards understanding the duality symmetries of other supersymmetric gauge and string theories [9]. It is now believed that the symmetries like S-duality are the symmetries of more genral string theories and can possibly help in handling their nonperturbative aspects. More recently, duality symmetries relating type II and heterotic string theories [2, 8] as well as the ones combining S- and T-duality into a bigger symmetry, namely U-duality [10], have also been discovered. The enlarged duality symmetries are also known to be present in dimensions other than four [10, 2, 6, 7]. For example, in three dimensions, the T-duality $O(7,23 ; Z)$ combines with the S-duality to form a bigger group $O(8,24 ; Z)[6$. In this paper, we study the duality symmetries of the two dimensional heterotic string theory by exploiting their resemblance to the Einstein-Maxwell theory with two commuting isometries.

Symmetries of the stationary axisymmetric Einstein-Maxwell equations have been studied a great deal over the years in the past [11, 12]. After the pioneering work by Geroch 13. for the stationary, axisymmetric Einstein equations, in a series of papers, Kinnersley et al[14, 15, 16, 17] unravelled a very rich symmetry structure in the stationary, axisymmetric Einstein-Maxwell equations, or equivalently, the so-called Ernst equations - a formulation due to Ernst. In fact, they showed that these equations enjoy an infinite-dimensional symmetry and found that the algebra is an infinite extension of the $\operatorname{sl}(3, R)$ algebra. It was

realized later that this was the current algebra $\hat{s l}(3, R)$ and it is this symmetry that renders 
the non-linear Ernst equations integrable. There exists an infinite number of conserved currrents in this theory and the algebra is realized in terms of them. A parallel of these studies has also been carried out for supergravity theories compactified to two dimensions. [18, 19]

Some aspects of the Ernst sigma model and the infinite dimensional symmetries have been analyzed recently in the context of heterotic string theory [20, 21, 22, 7]. In particular, the T- and S-duality symmetries of the four dimensional string theory in the presence of two commuting isometries have been shown to be a subgroup of the infinite dimensional String Geroch group [20, 7]. It was also shown in [7] that the $O(8,24)$ symmetry of the heterotic string theory in 3-dimensions [6] has an affinization to $\hat{O}(8,24)$ in 2-dimensions. However in these extensions to string theories, the emphasis has mainly been on the algebraic and symmetry structure and comparatively less on their applications to the classification and the generation of the background fields. More recently some work has been carried out to use these transformations for practical purposes in theories with axion and dilaton fields[24]. The full structure of the heterotic string theory, however, has been missing in these attempts since the moduli fields are not included.

In the approach by Kinnersley et, al[14, 15, 16, 17] to the investigation of the EinsteinMaxwell therory with two commuting isometries, both the algebraic structure of the symmetries as well as their applications to the gravity backgrounds are clarified. For example, the finite form of the symmetry transformations which can be used for generating solutions have been given in [14, 16]. They include interesting nontrivial transformations such as the Ehlers and the Harrison transformatins. The infinitesimal form of these transformations on an infinite set of potentials responsible for generating the affine algebra is also given in these papers.

In a previous paper 25, two of the present authors applied the formalism of Kinnersley et al to the studies of the string effective action and found a close analogy between the equations of motion of the Einstein gravity and the String effective action for vanishing 
$E_{8} \times E_{8}$ backgrounds. Based on this analogy, we gave the analogues of the Ehlers transformation for the string effective action and showed their connection to the $O(d, d)$ symmetry transformations. We in fact demonstrated that the generalized Ehlers transformation of string theory can be identified with the coset $\frac{O(d) \times O(d)}{O(d)}$ of the symmetry group $O(d, d)$. This $O(d, d)$, unlike T-duality, however acts on the variables $\psi, G$ and $\phi$, where $\psi$ is the field dual to the the antisymmetric moduli $B . G$ are the symmetric moduli and $\phi$ is the dilaton field. The action of the Ehlers transformation on $B$ is given by its induced action through a duality relation:

$$
\rho G^{-1} \partial_{\mu} B G^{-1}=-\epsilon_{\mu \nu} \partial^{\nu} \psi
$$

It was found that the $O(d, d)$ transformations can then be classified into three sets of $O(d, d)$ matrices satisying

$$
\Omega^{T} L \Omega=L, \quad L=\left(\begin{array}{cc}
0 & I_{d} \\
I_{d} & 0
\end{array}\right)
$$

as

$$
\Omega_{1}=\left(\begin{array}{cc}
I_{d} & \gamma \\
0 & I_{d}
\end{array}\right) \quad \Omega_{2}=\left(\begin{array}{cc}
I_{d} & 0 \\
\alpha & I_{d}
\end{array}\right) \quad \Omega_{3}=\left(\begin{array}{cc}
A^{-1^{T}} & 0 \\
0 & A
\end{array}\right),
$$

where $I_{d}$ is the $d$-dimensional identity matrix. Among these, $\Omega_{2}$ and $\Omega_{3}$ correspond to the constant shift in the antisymmetric moduli and the constant coordinate transformations respectively. $\Omega_{1}$ are the only transformations which act differently on $B$ and on $\psi$. They act non-locally on $B$ and are responsible for the presence of the infinite set of conserved currents. These are identified as the Ehlers transformation for string theory.

In this paper we extend the analysis of our previous work to include nontrivial gauge fields of heterotic string theory. We once again show a close analogy of the equations of motion of heterotic string theory with the analysis of [14, 15]. We generalize the finite dimensional groups $H^{\prime}$ and $G^{\prime}$ in their analysis of Einstein-Maxwell theory to the heterotic 
string theory. These groups in string case act manifestly on the original and the dual equations of motion respectively. We also explicitly construct the infinite number of conserved currents of this theory and their transformation rules starting from original as well as dual equations of motion. We identify the infinite dimensional symmetry algebra of this theory with the affine $\hat{o}(8,24)$ algebra and identify the analogues of the Ehlers and Harrison transformations. The duality symmetry of the two dimensional heterotic string theory is the discrete subgroup $\hat{O}(8,24 ; Z)$ of this infinite dimensional symmetry[0].

The rest of the paper is planned as follows. In section- 2 we give the equations of motion of the heterotic string and show their analogy with that of the Einstein-Maxwell equations. We also write down the equations of motions using the dual variables and show that they are also analogous to the equations in the Einstein-Maxwell theory. Section-3 gives the construction of the infinite set of potentials. The symmetry transformations, the related algebra and their identification with $\hat{o}(8,24)$ is given in section-4. These correspond to the enlargements of the $G^{\prime}$ and $H^{\prime}$ to the infinite dimensional group $K^{\prime}$ identified as $\hat{O}(8,24)$ in the present case. The conclusions and the discussions are given in section-5.

\section{Derivation of the Equations}

We now begin our study of the bosonic part of the two dimensional heterotic string theory with nontrivial dilaton and moduli fields which include the nontrivial $E_{8} \times E_{8}$ gauge field backgrounds of the ten dimensional heterotic string theory compactified to two dimensions. The situation is equivalent to considering the four dimensional heterotic string theory with two commuting isometries. Furthermore, since the gauge fields have no dynamics in two dimensions, the two dimensional gauge fields are chosen to have vanishing backgrounds 14, 7]. We shall start, therefore, with the 2-dimensional effective action of the heterotic string 
theory, namely,

$$
S=\int d^{2} x \sqrt{g} e^{-2 \phi}\left[R+4 g^{\mu \nu} \partial_{\mu} \phi \partial_{\nu} \phi+\frac{1}{8} g^{\mu \nu} \operatorname{Tr}\left(\partial_{\mu} \mathcal{M}^{-1} \partial_{\nu} \mathcal{M}\right)\right],
$$

where the matrix $\mathcal{M}$, representing the moduli $G, B$ and $A$ is parametrized as:

$$
\mathcal{M}=\left(\begin{array}{ccc}
G^{-1} & G^{-1}(B+C) & G^{-1} A \\
(-B+C) G^{-1} & (G-B+C) G^{-1}(G+B+C) & (G-B+C) G^{-1} A \\
A^{T} G^{-1} & A^{T} G^{-1}(G+B+C) & I_{16}+A^{T} G^{-1} A
\end{array}\right) .
$$

$G$ and $B$ are respectively $d \times d$ symmetric and antisymmetric matrix-valued scalar fields. $A$ denotes $d \times n$ matrix-valued scalar fields coming from the gauge fields of the heterotic strings. For the heterotic string theory, $d=8$ and $n=16$, but we keep these parameters arbitrary in the present discussion to avoid confusion among indices when they are used. $C=\frac{1}{2} A A^{T}$ is a $d \times d$ matrix. The equations of motion for the moduli $\mathcal{M}$ and dilaton $e^{-2 \phi}$ derived from the above action can be written as

$$
\begin{aligned}
\partial_{\mu}\left(\sqrt{g} g^{\mu \nu} e^{-2 \phi} \mathcal{M}^{-1} \partial_{\nu} \mathcal{M}\right) & =0, \\
\partial_{\mu}\left(\sqrt{g} g^{\mu \nu} \partial_{\nu} e^{-2 \phi}\right) & =0, \\
R_{\mu \nu}^{(2)}+2 \nabla_{\mu} \nabla_{\nu} \phi+\frac{1}{8} \operatorname{Tr}\left(\partial_{\mu} \mathcal{M}^{-1} \partial_{\nu} \mathcal{M}\right) & =0 .
\end{aligned}
$$

The equations (6) and (7) can be rewritten in the conformal gauge, with an identification $\rho=e^{-2 \phi}$, as

$$
\begin{aligned}
\partial^{\mu}\left(\rho \mathcal{M}^{-1} \partial_{\mu} \mathcal{M}\right) & =0 \\
\partial^{\mu} \partial_{\mu} \rho & =0
\end{aligned}
$$

$\mathcal{M}$ also has the properties:

$$
\mathcal{M}^{T}=\mathcal{M}, \quad \mathcal{L} \mathcal{M} \mathcal{L}=\mathcal{M}^{-1}
$$

where $L$ is given by

$$
\mathcal{L}=\left(\begin{array}{ccc}
0 & I_{d} & 0 \\
I_{d} & 0 & 0 \\
0 & 0 & I_{n}
\end{array}\right)
$$


and $I_{n}$ denotes an $n \times n$ identity matrix. The third equation (8) turns out to be an integrable one for the conformal factor and takes the following form in the conformal gauge

$$
\begin{aligned}
g_{\mu \nu}=e^{2 \Gamma} \delta_{\mu \nu}: & \\
-\delta_{\mu \nu} \partial^{\sigma} \partial_{\sigma} \Gamma+2\left(\partial_{\mu} \partial_{\nu} \phi\right. & \left.-\partial_{\mu} \phi \partial_{\nu} \Gamma-\partial_{\nu} \phi \partial_{\mu} \Gamma+\delta_{\mu \nu} \partial^{\sigma} \Gamma \partial_{\sigma} \phi\right) \\
& +\frac{1}{8} \operatorname{Tr}\left(\partial_{\mu} \mathcal{M}^{-1} \partial_{\nu} \mathcal{M}\right)=0 .
\end{aligned}
$$

Here we are choosing the two dimensional metric to be Euclidean. This corresponds to the contraction to the Levi-Civita tensor: $\epsilon^{\mu \alpha} \epsilon_{\mu \beta}=\delta_{\beta}^{\alpha}$. All the results, however, are valid in Minkowski space as well with few changes of signs in the formulas presented here. Determination of this conformal factor is important for the generation of new solutions. However, since this equation for $\Gamma$ is integrable, we only concentrate on equations (9), (10) for studying the symmetries of the theory. These equation are the same as that for the Ernst sigma model[11, 19, 20]. It is also clear that they are invariant under an $O(d, d+n)$ group of symmetry transformations:

$$
\mathcal{M} \rightarrow \tilde{\mathcal{M}}=\Omega \mathcal{M} \Omega^{T}
$$

where $\Omega$ satisfies

$$
\Omega^{T} \mathcal{L} \Omega=\mathcal{L}
$$

which includes the T-duality symmetry of the string theory as its discrete subgroup $O(d, d+$ $n ; Z)$ and has been used for generating solutions. The non-trivial action of T-duality is represented by a group $\frac{O(d ; Z) \times O(d+n ; Z)}{O(d ; Z)}[27$. The T-duality is also conjectured to be a symmetry, not only of the string effective action, but also of the full string theory[5]. However it has already been pointed out [7] that in two dimensions, which is the case at hand, the symmetry is not merely $O(8,24)$, but an infinite dimensional one, viz $\hat{O}(8,24)$. We shall show the existence of $\hat{O}(8,24)$ symmetry in later sections after dualizing equations (9) $-(10)$. 
At this point we would like to mention to the readers interested in seeing only the $\hat{O}(8,24)$ symmetry structure that they can directly proceed to the analysis of sections $(3 . b)$ and (4.b). However the full structure of the analysis by Kinnersley et al is unravelled only by studying the component forms of equation (9) and their dualizations. This is pursued in the following. The connection between the symmetry structures in the original and the dual formulations of supergravity also requires the full analysis of sections-3 and 4 . In section-5 we comment on the comparison between the dualization procedure given below and the one in section (3.b).

The symmetry transformations on the moduli is now determined on the lines of [14, 15] by obtaining the twist potentials. For this we observe that equations (9) are total divergence conditions and when written in terms of $G, B$, and $A$ take the form,

$$
\begin{aligned}
& \partial^{\mu}\left[\rho\left(\partial_{\mu} E^{T} G^{-1}+E^{T} G^{-1}\left(\partial_{\mu} B+\partial_{\mu} C-\partial_{\mu} A A^{T}\right) G^{-1}\right)\right]=0, \\
& \partial^{\mu}\left[\rho\left(E^{T} G^{-1} \partial_{\mu} E-\partial_{\mu} E^{T} G^{-1} E-E^{T} G^{-1}\left(\partial_{\mu} B+\partial_{\mu} C-\partial_{\mu} A A^{T}\right) G^{-1} E\right)\right]=0, \\
& \partial^{\mu}\left[\rho\left(E^{T} G^{-1} \partial_{\mu} A-\partial_{\mu} E^{T} G^{-1} A-E^{T} G^{-1}\left(\partial_{\mu} B+\partial_{\mu} C-\partial_{\mu} A A^{T}\right) G^{-1} A\right)\right]=0, \\
& \partial^{\mu}\left[\rho\left(G^{-1} \partial_{\mu} E-G^{-1}\left(\partial_{\mu} B+\partial_{\mu} C-\partial_{\mu} A A^{T}\right) G^{-1} E\right)\right]=0, \\
& \partial^{\mu}\left[\rho\left(G^{-1} \partial_{\mu} A-G^{-1}\left(\partial_{\mu} B+\partial_{\mu} C-\partial_{\mu} A A^{T}\right) G^{-1} A\right)\right]=0 \\
& \partial^{\mu}\left[\rho\left(A^{T} G^{-1} \partial_{\mu} A-\partial_{\mu} A^{T} G^{-1} A-A^{T} G^{-1}\left(\partial_{\mu} B+\partial_{\mu} C-\partial_{\mu} A A^{T}\right) G^{-1} A\right)\right]=0, \\
& \partial^{\mu}\left[\rho\left(G^{-1}\left(\partial_{\mu} B+\partial_{\mu} C-\partial_{\mu} A A^{T}\right) G^{-1}\right)\right]=0 \\
& \partial^{\mu}\left[\rho\left(\partial_{\mu} A^{T} G^{-1}+A^{T} G^{-1}\left(\partial_{\mu} B+\partial_{\mu} C-\partial_{\mu} A A^{T}\right) G^{-1}\right)\right]=0 \\
& \partial^{\mu}\left[\rho\left(A^{T} G^{-1} \partial_{\mu} E-\partial_{\mu} A^{T} G^{-1} E-A^{T} G^{-1}\left(\partial_{\mu} B+\partial_{\mu} C-\partial_{\mu} A A^{T}\right) G^{-1} E\right)\right]=0
\end{aligned}
$$

where $E \equiv G+B+C$, and superscript ${ }^{T}$ denotes the transpose. Note that these equations are not all independent. Equations (16), (18) and (20) are the transpose of equations (19), (24) and (23), respectively. This leaves us with six equations in three unknowns, viz. $G, B$ and $A$. However, they are also not all algebraically independent. For example, equation(18) 
is identically satisfied using (16) and (20). We shall take equations (19), (20) and (22) as three independent equations. The reduction to only three independent equations is due to the fact that eqn.(6) is basically the $O(d, d+n)$ invariant combination of the three equations of motion for $G, B$ and $A$. Equations (19) can be further simplified using the other two, so that the independent set of equations for the heterotic string theory can be written as:

$$
\begin{array}{r}
\partial^{\mu}\left[\rho G^{-1}\left(\partial_{\mu} B-\frac{1}{2} \partial_{\mu} A A^{T}+\frac{1}{2} A \partial_{\mu} A^{T}\right) G^{-1}\right]=0, \\
\partial^{\mu}\left(\rho G^{-1} \partial_{\mu} A\right)-\rho G^{-1}\left(\partial_{\mu} B-\frac{1}{2} \partial_{\mu} A A^{T}+\frac{1}{2} A \partial_{\mu} A^{T}\right) G^{-1} \partial^{\mu} A=0, \\
\partial^{\mu}\left(\rho G^{-1} \partial_{\mu} G\right)+\rho G^{-1} \partial_{\mu} A \partial^{\mu} A^{T} \\
+\rho G^{-1}\left(\partial_{\mu} B-\frac{1}{2} \partial_{\mu} A A^{T}+\frac{1}{2} A \partial_{\mu} A^{T}\right) G^{-1}\left(\partial^{\mu} B-\frac{1}{2} \partial^{\mu} A A^{T}+\frac{1}{2} A \partial^{\mu} A^{T}\right)=0 .
\end{array}
$$

The equations (25)-(27) are the matrix generalizations of the eqns.(7.7)-(7.10) of [14] with the identifications $A_{1}=B_{1}=\frac{A}{2}$ and $\psi_{11}+2 A_{1} B_{1} \equiv B$. We have thus shown a close relationship between the equations of motion of the heterotic string theory and the equations of the dual fields in the Einstein-Maxwell theory. These identifications allows us to apply the whole formulation of Kinnersley et al to the present problem with some variations. For example, unlike in [14, 15], in our case, the analogue of the component $A_{2}$ of the gauge field, namely $\tilde{A}$, does not represent a new degree of freedom.

We can now define two potentials $\psi$ and $\tilde{A}$ :

$$
\begin{aligned}
G^{-1}\left(\partial_{\mu} B+\partial_{\mu} C-\partial_{\mu} A A^{T}\right) G^{-1} & =\rho^{-1} \epsilon_{\mu \nu} \partial^{\nu} \psi \\
G^{-1} \partial_{\mu} A-G^{-1}\left(\partial_{\mu} B+\partial_{\mu} C-\partial_{\mu} A A^{T}\right) G^{-1} A & =\rho^{-1} \epsilon_{\mu \nu} \partial^{\nu}(\tilde{A}-\psi A),
\end{aligned}
$$

such that equations (25) and (26) are the integrability conditions for $\psi$ and $\tilde{A}$. One can also define another independent potential from equation (19), but we introduce it in the later sections.

Inserting $\tilde{A}$ and $\psi$ into the equations of motion one can rewrite them as divergence equations containing the dual potentials. By using (29) in itself once, one can write the 
following equations:

$$
\begin{aligned}
\epsilon_{\mu \nu} \partial^{\nu} \tilde{A} & =\left(\rho G^{-1}+\psi \frac{G}{\rho} \psi\right) \partial_{\mu} A-\psi \frac{G}{\rho} \partial_{\mu} \tilde{A} \\
\epsilon_{\mu \nu} \partial^{\nu} A & =-\frac{G}{\rho}\left(\partial_{\mu} \tilde{A}-\psi \partial_{\mu} A\right) .
\end{aligned}
$$

One can now use $G, \psi$ and $\rho$ to rewrite the equations (25)-(27). In this way one obtains a set of new equations:

$$
\begin{aligned}
\partial^{\mu}\left[\frac{G}{\rho}\left(\partial_{\mu} \tilde{A}-\psi \partial_{\mu} A\right)\right] & =0 \\
\partial^{\mu}\left[\rho G^{-1} \partial_{\mu} A-\psi \frac{G}{\rho}\left(\partial_{\mu} \tilde{A}-\psi \partial_{\mu} A\right)\right] & =0 \\
\partial^{\mu}\left[\frac{G}{\rho} \partial_{\mu} \psi G+\frac{G}{\rho}\left(\partial_{\mu} \tilde{A}-\psi \partial_{\mu} A\right) A^{T}\right] & =0 \\
\partial^{\mu}\left(\rho G^{-1} \partial_{\mu} G\right)-\partial^{\mu} \psi \frac{G}{\rho} \partial_{\mu} \psi G+\rho G^{-1} \partial_{\mu} A \partial^{\mu} A^{T} & =0 .
\end{aligned}
$$

These are once again of the same form as the equations in the Einstein-Maxwell theory. For example, the form of our eqns.(32)-(35) match with those of (7.1)-(7.4) of [14] with the identifications $A_{1} \equiv \frac{A}{2}, A_{2} \equiv \frac{\tilde{A}}{2}$ and $\omega \equiv \psi$. Other equations, namely the analogues of (7.5) and (7.6) in [14, are derivable from the above ones and have the same form.

At this point we note a major difference between the situations in the Einstein-Maxwell theory and string case. We find that the roles of the original and dual variables as compared to [14, 15, 16] have changed in our case. Namely the $B$ field of string theory plays the role of the dual variable $\psi_{11}$ in $[14]$ and our $\psi$ plays the role of the twist potential $\omega$ parameterizing the metric in [14]. As a result the Ehlers and the Harrison transformations as written in [14] can not be directly copied to our case, since they will simply correspond to our T-duality transformations in equations (14). Our task, on the other hand, is to find the symmetry transformations with non-local action on $B$.

One can also combine the two equations (30) and (31) into a single one:

$$
\partial_{\mu} \Phi=-M L \epsilon_{\mu \nu} \partial^{\nu} \Phi
$$


where $M$ is a $2 d \times 2 d$ matrix

$$
M=\left(\begin{array}{cc}
-\rho G^{-1}-\psi \frac{G}{\rho} \psi & \psi \frac{G}{\rho} \\
-\frac{G}{\rho} \psi & \frac{G}{\rho}
\end{array}\right),
$$

with $L M L=-M^{-1}$ and $\Phi$ is an $n \times n$ matrix written as

$$
\Phi=\left(\begin{array}{c}
\tilde{A} \\
A
\end{array}\right) .
$$

Equation (36) also implies

$$
\partial^{\mu}\left(M^{-1} \partial_{\mu} \Phi\right)=0,
$$

and is equivalent to the two equations (32) and (33). Similarly the two equations (34) and (35) can be combined as

$$
\partial^{\mu}\left[\rho M^{-1} \partial_{\mu} M-M^{-1} \partial_{\mu} \Phi \Phi^{T}\right]=0 .
$$

Thus, starting from our original set of equations of motion, we have arrived at a formulation

of the equations that consists in the variables $G, \psi, A$, and $\tilde{A}$. Unlike $B, A$ has not been eliminated in favour of its dual in equations of motion. Setting the gauge fields to zero we also retrieve the expression for $M$ as well as the equations of motion of the dual fields given in 25. .

It can be shown [29] that the dualized equations of motion (32)-(35) naturally follow from the dimensional compactification of the $N=1$ supergravity- super-Yang-Mills action in ten dimensions [5, 30] down to two. We now study the symmetries of the equations (39), (40) as well as the original equations (9)-(10).

\section{Symmetries of the equations of motion}

We observe that the two equations (39)-(40) are manifestly invaraint under the following transformations:

$$
(i) M \rightarrow \Omega M \Omega^{T}, \quad \Phi \rightarrow \Omega \Phi, \quad \Omega^{T} L \Omega=L
$$




$$
\begin{array}{r}
(i i) M \rightarrow M, \quad \Phi \rightarrow \Phi \Omega^{T}, \quad \Omega^{T} \Omega=I_{n} \\
(\text { iii }) M \rightarrow M, \quad \Phi \rightarrow \Phi+\text { constt } .
\end{array}
$$

These transformations are the extensions of the group $G^{\prime}$ mentioned in [14. Similarly the $O(8,24)$ mentioned in equation (14) is the analogue of the group $H^{\prime}$ of [14]. Among $G^{\prime}$ symmetries, (i) and (iii) are the same transformations mentioned in [14]. (ii) is a new symmetry for us, since $A$ in our case represents $n$-columns as opposed to a single function $A_{1}$ or $A_{2}$ in [14. Together, these are a 496-parameter global symmetry transformations of the dual equations of motion for the heterotic strings. Note that although equations (39) and (40) are not manifestly invariant under $O(8,24)$, we will still be able to show that these equations have an affine $\hat{O}(8,24)$ symmetry and identify $G^{\prime}$ as part of this.

In subsection (3.a) below we now give the construction of the infinite set of conserved currents starting from the dual equations (39)- (40). The same exercise for the original field equations (9)- (10) is done in section (3.b).

Dealing with the dual equations of motion (32)- (35) is technically more complicated than the original equations of motion. This is essentially due to the fact that the manifest symmetries in the dimensional compactification of the dual formulation of the supergravitysuper-Yang-Mills are different from the original formulation. This has previously been observed in four dimensions [5, 30]. In our case the manifest symmetry is only and $O(8,8)$ symmetry. We however show that the full symmetry in this case is also an affine $\hat{O}(8,24)$ symmetry.

\section{(3.a) Conserved Currents from the Dual Equations of Motion}

The basic property needed for generating the infinite dimensional algebra using the procedure of [14, 15, 16] is to find a set of variables satisfying the Ernst equations. The $\Phi$ in equation (36) already satifies this property. To find other quantities which satisfy similar 
equations, we introduce a potential $\Psi$ as

$$
M^{-1} \partial_{\mu}(\rho M)-M^{-1} \partial_{\mu} \Phi \Phi^{T}=-\epsilon_{\mu \nu} \partial^{\nu} \Psi,
$$

which also implies

$$
M\left(\partial_{\mu} \Psi+L \partial_{\mu} \Phi \Phi^{T}\right)=\epsilon_{\mu \nu} \partial^{\nu}(\rho M),
$$

and is equivalent to the equation of motion (40). Then $\mathcal{H}$ defined as $\mathcal{H} \equiv \rho M+L \Psi$, satisfies the Ernst equation,

$$
\partial_{\mu} \mathcal{H}=-M L \epsilon_{\mu \nu} \partial^{\nu} \mathcal{H}
$$

One can now use $\Phi$ and $\mathcal{H}$ to construct the infinite hierarchy of potentials in terms of which the infinite dimensional symmetry algebra will be realized. Let us note at this point that both $\mathcal{H}$ and $\Phi$ contain the mixture of original and dual fields. This mixture of fields is also crucial for the generation of new solutions. Treating $\mathcal{H}$ and $\Phi$ as our field variables, four bilinear potentials are defined through the following relations:

$$
\begin{aligned}
\partial_{\mu} \mathcal{K} & =\Phi^{T} L \partial_{\mu} \Phi, \\
\partial_{\mu} \mathcal{N} & =\mathcal{H}^{T} L \partial_{\mu} \mathcal{H}, \\
\partial_{\mu} \mathcal{Q} & =\Phi^{T} L \partial_{\mu} \mathcal{H}, \\
\partial_{\mu} \mathcal{P} & =\mathcal{H}^{T} L \partial_{\mu} \Phi .
\end{aligned}
$$

It can be verified, using (36) and (46), that they satisfy the condition:

$$
\epsilon_{\mu \nu} \partial^{\nu} \partial^{\mu} \mathcal{K}=\epsilon_{\mu \nu} \partial^{\nu} \partial^{\mu} \mathcal{N}=\epsilon_{\mu \nu} \partial^{\nu} \partial^{\mu} \mathcal{Q}=\epsilon_{\mu \nu} \partial^{\nu} \partial^{\mu} \mathcal{P}=0 .
$$

Furthermore one can define more fields out of the four potentials $\mathcal{K}, \mathcal{N}, \mathcal{Q}$ and $\mathcal{P}$ which also satisfy the Ernst equations. For example the fields $\mathcal{R}$ and $\mathcal{U}$ defined as

$$
\begin{gathered}
\mathcal{U}=\mathcal{N}+\Phi \mathcal{Q}+\mathcal{H} L \mathcal{H} \\
\mathcal{R}=\mathcal{P}+\Phi \mathcal{K}+\mathcal{H} L \Phi
\end{gathered}
$$


satisfy field equations similar to those of $\mathcal{H}$ and $\Phi$, respectively, viz.

$$
\begin{aligned}
\partial_{\mu} \mathcal{R} & =-M L \epsilon_{\mu \nu} \partial^{\nu} \mathcal{R} \\
\partial_{\mu} \mathcal{U} & =-M L \epsilon_{\mu \nu} \partial^{\nu} \mathcal{U} .
\end{aligned}
$$

In deriving the equations (54) and (55) for $\mathcal{R}$ and $\mathcal{U}$, we had to use an identity

$$
\partial_{\mu}\left(L \Psi+\Psi^{T} L+\Phi \Phi^{T}\right)=-2 \partial_{\mu} z L,
$$

where $\epsilon_{\mu \nu} \partial^{\nu} z=\partial_{\mu} \rho . \mathcal{R}$ and $\mathcal{U}$ can now be used in place of $\Phi$ and $\mathcal{H}$ to define a second set of potentials as in (47)-(50) and thence another set of fields, as in (52)-(53) and so on, each time increasing the order of fields in the potentials by one. This bestowes us with an infinite set of fields $\left\{\mathcal{H}^{n} \mid n=1,2 \cdots\right\}$ and $\left\{\Phi^{n} \mid n=1,2 \cdots\right\}$ with

$$
\mathcal{H}^{1}=\mathcal{H} \quad \text { and } \quad \Phi^{1}=\Phi,
$$

each satifying the same field equations:

$$
\begin{gathered}
\partial_{\mu} \mathcal{H}^{n}=-M L \epsilon_{\mu \nu} \partial^{\nu} \mathcal{H}^{n}, \\
\partial_{\mu} \Phi^{n}=-M L \epsilon_{\mu \nu} \partial^{\nu} \Phi^{n} .
\end{gathered}
$$

From these fields we can once again define four families of potentials as [cf. (47) and (50) ]

$$
\begin{gathered}
\partial_{\mu} \mathcal{K}^{m n}=\Phi^{T m} L \partial_{\mu} \Phi^{n}, \\
\partial_{\mu} \mathcal{N}^{m n}=\mathcal{H}^{T m} L \partial_{\mu} \mathcal{H}^{n}, \\
\partial_{\mu} \mathcal{Q}^{m n}=\Phi^{T m} L \partial_{\mu} \mathcal{H}^{n}, \\
\partial_{\mu} \mathcal{P}^{m n}=\mathcal{H}^{T m} L \partial_{\mu} \Phi^{n},
\end{gathered}
$$

which satisfy equations similar to (51). These in turn enable us to define the solutions of the field equations cf. (36)-(46):

$$
\begin{gathered}
\mathcal{H}^{n+1}=\mathcal{N}^{1 n}+\Phi \mathcal{Q}^{1 n}+\mathcal{H} L \mathcal{H}^{n}, \\
\Phi^{n+1}=\mathcal{P}^{1 n}+\Phi \mathcal{K}^{1 n}+\mathcal{H} L \Phi^{n},
\end{gathered}
$$


as the $(n+1)$-th set of fields from the $n$-th set in the hierarchy. Due to the matrix-valuedness of $A$ in our case, $\mathcal{K}$ for us is an $n \times n$ matrix instead of being a single number as in [14, 15]. $\mathcal{P}$ and $\mathcal{Q}$ are respectively $2 d \times n$ and $n \times 2 d$ matrices and $\mathcal{N}$ is a $2 d \times 2 d$ matrix.

Several properties of the potentials follow from their definitions,

$$
\begin{gathered}
\mathcal{K}^{m n}+\mathcal{K}^{T n m}=\Phi^{T m} L \Phi^{n}, \\
\mathcal{Q}^{m n}+\mathcal{P}^{T n m}=\Phi^{T m} L \mathcal{H}^{n}, \\
\mathcal{N}^{m n}+\mathcal{N}^{T n m}=\mathcal{H}^{T m} L \mathcal{H}^{n} .
\end{gathered}
$$

Moreover, the potentials are interrelated by some identities, namely,

$$
\begin{aligned}
\mathcal{K}^{m, n+1}-\mathcal{K}^{m+1, n} & =\mathcal{Q}^{m 1} L \Phi^{n}+\mathcal{K}^{m 1} \mathcal{K}^{1 n}, \\
\mathcal{Q}^{m, n+1}-\mathcal{Q}^{m+1, n} & =\mathcal{Q}^{m 1} L \mathcal{H}^{n}+\mathcal{K}^{m 1} \mathcal{Q}^{1 n}, \\
\mathcal{P}^{m, n+1}-\mathcal{P}^{m+1, n} & =\mathcal{N}^{m 1} L \Phi^{n}+\mathcal{P}^{m 1} \mathcal{K}^{1 n}, \\
\mathcal{N}^{m, n+1}-\mathcal{N}^{m+1, n} & =\mathcal{N}^{m 1} L \mathcal{H}^{n}+\mathcal{P}^{m 1} \mathcal{Q}^{1 n}
\end{aligned}
$$

Derivation of equations (66)-(72) involve integration by parts. We can set the constants of integrations to zero without any loss of generality. For later convenience, we shall now define the potentials for all, i.e., positive, zero and negative integer, values of $m$ and $n$. Let us define

$$
\mathcal{K}^{10}=I_{n}, \quad \mathcal{H}^{0}=L .
$$

Then, from the definitions of $\mathcal{P}^{m n}$ and $\mathcal{N}^{m n}$, equations (63) and (61), one can identify

$$
\mathcal{P}^{0 n}=\Phi^{n} \quad \text { and } \quad \mathcal{N}^{0 n}=\mathcal{H}^{n} .
$$

We shall also define

$$
\mathcal{K}^{1-p, p}=-\mathcal{K}^{p, 1-p}=-I_{n} \quad \text { and } \quad \mathcal{N}^{p,-p}=-\mathcal{N}^{-p, p}=L,
$$


where $p \geq 1$. All other quantities with $m, n \leq 0$ are defined to vanish. One can now generalize the recursion relations (69) $-(\sqrt[72)]{ })$ to read, respectively, as:

$$
\begin{aligned}
\sum_{s}\left[\mathcal{Q}^{m s} L \mathcal{P}^{1-s, n}+\mathcal{K}^{m s} \mathcal{K}^{2-s, n}\right] & =0, \\
\sum_{s}\left[\mathcal{Q}^{m s} L \mathcal{N}^{1-s, n}+\mathcal{K}^{m s} \mathcal{Q}^{2-s, n}\right] & =0, \\
\sum_{s}\left[\mathcal{N}^{m s} L \mathcal{P}^{1-s, n}+\mathcal{P}^{m s} \mathcal{K}^{2-s, n}\right] & =0, \\
\sum_{s}\left[\mathcal{N}^{m s} L \mathcal{N}^{1-s, n}+\mathcal{P}^{m s} \mathcal{Q}^{2-s, n}\right] & =0,
\end{aligned}
$$

where $s$ runs over all integers, $-\infty \leq s \leq+\infty$.

We have thus shown the presence of the four sets of infinite number of conserved currents in our two dimensional theory corresponding to the potentials $\mathcal{K}, \mathcal{P}, \mathcal{Q}$ and $\mathcal{N}$. In the next section we find the symmetry transformations corresponding to these infinite set of conserved currents and the corresponding algebra. Before that however we give the same construction of currents starting with the field equation (9).

\section{(3.b) Currents from the Original Equations of Motion}

By starting with the original field equations (9), one can define a potential:

$$
\mathcal{M}^{-1} \partial_{\mu}(\rho \mathcal{M})=-\epsilon_{\mu \nu} \partial^{\nu} \hat{\Psi} .
$$

Then $\hat{\mathcal{H}} \equiv \rho \mathcal{M}+i \mathcal{L} \hat{\Psi}$ also satisfies the Ernst equation:

$$
\partial_{\mu} \hat{\mathcal{H}}=i \mathcal{M L} \epsilon_{\mu \nu} \partial^{\nu} \hat{\mathcal{H}} .
$$

One can therefore obtain the currents on the same line as in the previous subsection. We now have

$$
\partial_{\mu} \hat{\mathcal{N}}=\hat{\mathcal{H}}^{T} \mathcal{L} \partial_{\mu} \hat{\mathcal{H}}
$$

which is now a $(2 d+n) \times(2 d+n)$ matrix and satisfies,

$$
\epsilon_{\mu \nu} \partial^{\nu} \partial^{\mu} \hat{\mathcal{N}}=0 .
$$


Similarly by using the identity,

$$
\partial_{\mu}\left(\mathcal{L} \hat{\Psi}+\hat{\Psi}^{T} \mathcal{L}\right)=-2 \partial_{\mu} z \mathcal{L}
$$

one finds that $\hat{\mathcal{U}}=\hat{\mathcal{N}}+\hat{\mathcal{H}} \mathcal{L} \hat{\mathcal{H}}$ also satisfies

$$
\partial_{\mu} \hat{\mathcal{U}}=i \mathcal{M} \mathcal{L} \epsilon_{\mu \nu} \partial^{\nu} \hat{\mathcal{U}}
$$

Repeating this process, one gets an infinite set of fields $\left\{\hat{\mathcal{H}}^{n} \mid n=1,2 \ldots\right\}$ with $\hat{\mathcal{H}}^{1}=\hat{\mathcal{H}}$ which satisfy the same field equations:

$$
\partial_{\mu} \hat{\mathcal{H}}^{n}=i \mathcal{M L} \epsilon_{\mu \nu} \partial^{\nu} \hat{\mathcal{H}}^{n}
$$

One also has a family of potentials represented by $(2 d+n) \times(2 d+n)$ matrices:

$$
\partial_{\mu} \hat{\mathcal{N}}^{m n}=\hat{\mathcal{H}}^{T m} \mathcal{L} \partial_{\mu} \hat{\mathcal{H}}^{n}
$$

which satisfy equations similar to (83). This in turn enables us to define the solutions of the field equations as:

$$
\hat{\mathcal{H}}^{n+1}=\hat{\mathcal{N}}^{1 n}+\hat{\mathcal{H}} \mathcal{L} \hat{\mathcal{H}}^{n}
$$

$\hat{\mathcal{N}}^{m n}$ satisfy the identities, $\hat{\mathcal{N}}^{m n}+\hat{\mathcal{N}}^{T n m}=\hat{\mathcal{H}}^{T m} \mathcal{L} \hat{\mathcal{H}}^{n}$ and are interrelated by

$$
\hat{\mathcal{N}}^{m, n+1}-\hat{\mathcal{N}}^{m+1, n}=\hat{\mathcal{N}}^{m 1} \mathcal{L} \hat{\mathcal{H}}^{n}
$$

Once again potential for all, i.e., positive, zero and negative integer values of $m$ and $n$ are defined by setting $\hat{\mathcal{H}}^{0}=\mathcal{L}$ which also implies $\hat{\mathcal{N}}^{0 n}=\hat{\mathcal{H}}^{n}$. One also sets $\hat{\mathcal{N}}^{p,-p}=-\hat{\mathcal{N}}^{-p, p}=$ $\mathcal{L}$, where $p \geq 1$. All other quanitities with $m, n \leq 0$ are again defined to vanish. One can then again generalize the recursion relations (89) to

$$
\sum_{s}\left[\hat{\mathcal{N}}^{m s} \mathcal{L} \hat{\mathcal{N}}^{1-s, n}\right]=0
$$

where $s$ runs over all integers, $-\infty \leq s \leq+\infty$. 


\section{Symmetry algebra}

In this section we first construct the $\hat{o}(8,24)$ algebra corresponding to the currents of section-(3.a) and later on those corresponding to the currents in section-(3.b).

\section{(4.a) Algebra from the Dual Equations}

Let us now note that the equations $(\sqrt{76})-(79)$ are invariant under three sets of transformations, whose parameters will be denoted by $\gamma^{k}$, $\xi^{k}$ and $\sigma^{k}$. These are respectively $2 d \times 2 d, n \times 2 d$ and $n \times n$ matrices and are the analogues of $\gamma^{k}, c^{k}$ and $\sigma^{k}$ of [14, 15]. It can be checked that the equations $(\sqrt{76})-(79)$ are invariant under the following set of transformations acting on variables defined in section-(3.a):

$$
\begin{aligned}
(i) \gamma^{m n}: \quad \mathcal{K}^{m n} & \rightarrow \mathcal{K}^{m n}-\sum_{i=1}^{k} \mathcal{Q}^{m i} L \gamma^{k} L P^{k-i, n} \\
\mathcal{Q}^{m n} & \rightarrow \mathcal{Q}^{m n}+\mathcal{Q}^{m, n+k} L \gamma^{k}-\sum_{i=1}^{k} \mathcal{Q}^{m i} L \gamma^{k} L \mathcal{N}^{k-i, n} \\
\mathcal{P}^{m n} & \rightarrow \mathcal{P}^{m n}-\gamma^{k} L \mathcal{P}^{m+k, n}-\sum_{i=1}^{k} \mathcal{N}^{m i} L \gamma^{k} L \mathcal{P}^{k-i, n} \\
\mathcal{N}^{m n} & \rightarrow \mathcal{N}^{m n}-\gamma^{k} L \mathcal{N}^{m+k, n}+\mathcal{N}^{m, n+k} L \gamma^{k}-\sum_{i=1}^{k} \mathcal{N}^{m i} L \gamma^{k} L \mathcal{N}^{k-i, n}
\end{aligned}
$$

where $\gamma^{k}$ is a $2 d \times 2 d$ antisymmetric matrix.

$$
\begin{aligned}
(i i) \xi^{k}: \quad \mathcal{K}^{m n} & \rightarrow \mathcal{K}^{m n}-\xi^{k} L \mathcal{P}^{m+k-1, n}-\mathcal{Q}^{m, n+k-1} L \xi^{T k}-\sum_{i=1}^{k} \mathcal{K}^{m i} \xi^{k} L \mathcal{P}^{k-i, n} \\
& +\sum_{i=1}^{k-1} \mathcal{Q}^{m i} L \xi^{T k} \mathcal{K}^{k-i, n} \\
\mathcal{Q}^{m n} & \rightarrow \mathcal{Q}^{m n}-\xi^{k} L \mathcal{N}^{m+k-1, n}+\mathcal{K}^{m, n+k} \xi^{k}-\sum_{i=1}^{k} \mathcal{K}^{m i} \xi^{k} L \mathcal{N}^{k-i, n} \\
& +\sum_{i=1}^{k-1} \mathcal{Q}^{m i} L \xi^{T k} \mathcal{Q}^{k-i, n}, \\
\mathcal{P}^{m n} & \rightarrow \mathcal{P}^{m n}+\xi^{T k} \mathcal{K}^{m+k, n}-\mathcal{N}^{m, n+k-1} L \xi^{T k}-\sum_{i=1}^{k} \mathcal{P}^{m i} \xi^{k} L \mathcal{P}^{k-i, n}
\end{aligned}
$$




$$
\begin{aligned}
& +\sum_{i=1}^{k-1} \mathcal{N}^{m i} L \xi^{T k} \mathcal{K}^{k-i, n} \\
\mathcal{N}^{m n} & \rightarrow \mathcal{N}^{m n}+\mathcal{P}^{m, n+k} \xi^{k}+\xi^{T k} \mathcal{Q}^{m+k, n}-\sum_{i=1}^{k} \mathcal{P}^{m i} \xi^{k} L \mathcal{N}^{k-i, n} \\
& +\sum_{i=1}^{k-1} \mathcal{N}^{m i} L \xi^{T k} \mathcal{Q}^{k-i, n}
\end{aligned}
$$

where $\xi^{k}$ is a $2 d \times n$ real matrix.

$$
\begin{aligned}
(i i i) \sigma^{k}: \quad \mathcal{K}^{m n} & \rightarrow \mathcal{K}^{m n}-\sigma^{k} \mathcal{K}^{m+k, n}+\mathcal{K}^{m, n+k} \sigma^{k}-\sum_{i=1}^{k} \mathcal{K}^{m i} \sigma^{k} \mathcal{K}^{k-i+1, n} \\
\mathcal{Q}^{m n} & \rightarrow \mathcal{Q}^{m n}-\sigma^{k} \mathcal{Q}^{m+k, n}-\sum_{i=1}^{k} \mathcal{K}^{m i} \sigma^{k} \mathcal{Q}^{k-i+1, n} \\
\mathcal{P}^{m n} & \rightarrow \mathcal{P}^{m n}+\mathcal{P}^{m, n+k} \sigma^{k}-\sum_{i=1}^{k} \mathcal{P}^{m i} \sigma^{k} \mathcal{K}^{k-i+1, n} \\
\mathcal{N}^{m n} & \rightarrow \mathcal{N}^{m n}-\sum_{i=1}^{k} \mathcal{P}^{m i} \sigma^{k} \mathcal{Q}^{k-i+1, n}
\end{aligned}
$$

where $\sigma^{k}$ is an $n \times n$ antisymmetric matrix.

It can be verified that the transformations (91)-(102) keep the recursion relations (76)(79) as well as the Ernst equations invariant and therefore generate solutions of (36) and (46) through these recursions. To show the invariance of the Ernst equations, one needs to integrate the identity (56) with vanishing integration constant.

The symmetry algebra can be obtained by using the transformation rules given above through their commutators. We get after some algebra:

$$
\begin{aligned}
{\left[\delta \sigma^{k}, \delta \sigma^{l}\right]=\delta \sigma^{k+l} } & , & \sigma^{k+l}=\left(\sigma^{k} \sigma^{l}-\sigma^{l} \sigma^{k}\right) \\
{\left[\delta \sigma^{k}, \delta \gamma^{l}\right]=0 } & & \\
{\left[\delta \sigma^{k}, \delta \xi^{l}\right]=\delta \xi^{k+l} } & , & \xi^{k+l}=\sigma^{k} \xi^{l} \\
{\left[\delta \gamma^{k}, \delta \gamma^{l}\right]=\delta \gamma^{k+l} } & , & \gamma^{k+l}=\left(\gamma^{k} L \gamma^{l}-\gamma^{l} L \gamma^{k}\right) \\
{\left[\delta \xi^{k}, \delta \gamma^{l}\right]=\delta \xi^{k+l} } & , & \xi^{k+l}=\xi^{k} L \gamma^{l}
\end{aligned}
$$




$$
\begin{aligned}
{\left[\delta \xi^{k}, \delta \xi^{l}\right]=\delta \gamma^{k+l-1}+\delta \sigma^{k+l-1} \quad, \quad \gamma^{k+l-1} } & =\left(\xi^{T k} \xi^{l}-\xi^{T l} \xi^{k}\right) \\
\text { and } \quad \sigma^{k+l-1} & =\left(\xi^{k} L \xi^{T l}-\xi^{l} L \xi^{T k}\right) .
\end{aligned}
$$

One can also write this symmetry algebra in a compact form by introducing a set of $2 d+n$ dimensional antisymmetric matrices generating the symmetry transformations:

$$
\Gamma_{1}^{k}=\left(\begin{array}{cc}
\gamma^{k} & 0 \\
0 & 0
\end{array}\right) \quad \Gamma_{2}^{k}=\left(\begin{array}{cc}
0 & 0 \\
0 & \sigma^{k}
\end{array}\right) \quad \Gamma_{3}^{k}=\left(\begin{array}{cc}
0 & \xi^{k^{T}} \\
-\xi^{k} & 0
\end{array}\right)
$$

It can then be shown that the algebra in equations (103)-(108) combine into

$$
\left[\delta \Gamma^{k}, \delta \Gamma^{l}\right]=\delta \Gamma^{k+l}
$$

where $\Gamma^{k+l}=\Gamma^{k} \mathcal{L} \Gamma^{l}-\Gamma^{l} \mathcal{L} \Gamma^{k}$.

One can identify the $\Gamma_{a}^{k}$ 's in eqn.(109) as the generators of the $O(8,24)$ transformations and therefore equation (110) represents an affine $\hat{o}(8,24)$ symmetry algebra.

\section{(4.b) Algebra from the Original Equations}

The symmetry transformations of the infinite set of potentials $\hat{\mathcal{N}}^{m n}$ as constructed in section (3.b) from the field equation (9) is given by

$$
\hat{\mathcal{N}}^{m n} \rightarrow \hat{\mathcal{N}}^{m n}-\hat{\Gamma}^{k} \mathcal{L} \hat{\mathcal{N}}^{m+k, n}+\hat{\mathcal{N}}^{m, n+k} \mathcal{L} \hat{\Gamma}^{k}-\sum_{i=1}^{k} \hat{\mathcal{N}}^{m i} \mathcal{L} \hat{\Gamma}^{k} \mathcal{L} \hat{\mathcal{N}}^{k-i, n}
$$

where $\hat{\Gamma}^{k}$ is a $(2 d+n) \times(2 d+n)$ antisymmetric matrix. Once again we have verified that these transformations leave the recursion relations, as well as the field equations, invariant. In this case one directly gets the $\hat{o}(8,24)$ symmetry algebra:

$$
\left[\delta \hat{\Gamma}^{k}, \delta \hat{\Gamma}^{l}\right]=\delta \hat{\Gamma}^{k+l}
$$

where $\hat{\Gamma}^{k+l}=\hat{\Gamma}^{k} \mathcal{L} \hat{\Gamma}^{l}-\hat{\Gamma}^{l} \mathcal{L} \hat{\Gamma}^{k}$ 


\section{Discussions}

In this paper we have shown the connection of the two dimensional heterotic string equations of motion and its symmetries with those of the axisymmetrix Einstein-Maxwell equa-

tions. The existence of an infinite number of conserved currents and $\hat{O}(8,24)$ symmetry in the two dimensional heterotic string theory has also been shown by using this analogy. The construction of the infinite symmetry structure was given for both the original equations of motion of the heterotic string as well as the one written after dualization and the elimination of the field $B$. The construction of the $\hat{o}(8,24)$ for the dual equations of motion was possible, even though they are not manifestly invariant under an $O(8,24)$ symmetry group. As already mentioned, these dual equations of motion have a natural interpretation in terms of the compactification of the dual $N=1$ supergravity-super Yang-Mills action in ten dimensions. Our results therefore imply a Geroch symmetry in the dual formulation as well. As expected, due to the absence of a manifest $O(8,24)$ symmetry in the equations of motion, the algebraic complications for this case in sections- (3.a) and (4.a) turned out to be considerably more than for the original equations of motion.

In the present procedure, the action of the symmetry transformations on our original fields in equation (司) can also be found. This can be done for the symmetry transformations in section-(4.a) by identifying $\Psi_{11}$ as $B$ through the dualization relations. Other fields, $G$ and $A$ already appear in the transformation laws through $\Phi$ and $M$. For example, The transforamtion by the parameters $\gamma^{0}$ is simply the infinitesimal version of the $O(d, d)$ transformations in equation (41). This can be seen by writing down its action on the potentials. They can be shown to transform as

$$
\mathcal{N} \rightarrow \Omega \mathcal{N} \Omega^{T}, \quad \mathcal{P} \rightarrow \Omega \mathcal{P}, \quad \mathcal{Q} \rightarrow \mathcal{Q} \Omega^{T}, \quad \text { and } \quad \mathcal{K} \rightarrow \mathcal{K}
$$

The infitesimal form of these transformations matches with (91)-(94), since all the quadratic 
terms in these equations vanish for $k=0$. Similarly it can be shown that the transformations (42) correspond to $\sigma^{0}$ and (43) to $c^{0}$.

The nontrivial action of $\gamma^{0}$ is given by the Ehlers transformation whch is represented by $\Omega$ of the form $\Omega_{2}$ in equation (3). The situation here is different from [14, 15] where the Ehlers transformation is represented by $\gamma^{1}$. This is because, unlike in [14, 15] our $M$ already represents the dual variables and a linear transformation like $\gamma^{0}$ induces non-local transformation of $B$. The action of these transformations on $B$ can also be found out explicitly, but we do not give them here. For vanishing $A, \tilde{A}$ backgrounds, the action of $\gamma^{0}$, or equivalently the transformations of equation (41) reduces to the Ehlers transformation in 251 .

On the other hand, if one proceeds by using the results of our sections-(3.b) and (4.b), namely the infinite extensions of the original equations of motion, then the Ehlers as well as the Harrison transformations are identified as the $\hat{\Gamma}^{1}$ transformations. In this case $\hat{\Gamma}^{0}$ is simply the T-duality rotations of equation (14).

We now discuss the connection between the two sets of conserved currents as well as symmetry structure shown in sections (3.a)-(4.a) and (3.b)-(4.b). For the later one, the $O(8,24)$ symmetry is manifest, whereas for the former ones this has been shown only after studying the algebraic structure. It may be possible in the former case also to combine the fields appearing in the dual equations of motion into a manifestly $O(8,24)$ invariant form. However it probably requires the elimination of one of the degree of freedom out of $A$ and $\tilde{A}$. This can be done possibly by finding a gauge symmetry and then by making a proper choice of gauge. It may be interesting to study this aspect further.

In this context, it is also important to find out the connection between the dual variables $\hat{\Psi}$, which appears as the dual of $\mathcal{M}$ and the matrices $M$ and $\Phi$ which appear in the dual set of equations of motion. By comparing equations (28), (29) with the components of (80) one recoginizes $\hat{\Psi}_{21} \equiv \psi$ and $\hat{\Psi}_{23} \equiv(\tilde{A}-\psi A)$. However a complete identification of the 
components of $\hat{\Psi}$ with those of $M$ and $\Phi$ involves potentials other than the two mentioned above. Similar problem also occurs in the identification of $\Psi$ in terms of the fileds $G, B$ and $A$.

It has been pointed out in [14 that the infinite dimensional symmetry $K^{\prime}$ identified as $\hat{s} l(3, R)$, which is the analogue of $\hat{O}(8,24)$ in our case, can be obtained by the combined operations of finite dimensional symmetries, $G^{\prime}$ and $H^{\prime}$. Extensions to infinite dimensional symmetry by the combined operations of T- and S-duality has also been noted recently [26]. It will therefore be interesting to identify $G^{\prime}$ as a combination of T- and S-duality. In this context we have already identified $H^{\prime}$ as the T-duality symmetry of the two dimensional heterotic string.

There are many other interesting avenues to explore in this area. First, one should be able to write down the finite action of all the transformations $\gamma^{k}, \xi^{k}, \sigma^{k}$ and $\hat{\Gamma}^{k}$ rather explicitly by following the later works of Kinnersley et al. In particular the exponentiation of $\hat{\Gamma}^{1}$ will give the finite form of the Ehlers and Harrison transformations. This will be important for generating the solutions of the heterotic strings similar to the EinsteinMaxwell case 28.

In the light of recent progress made in understanding the duality symmetries of the $N=2$ supersymmetric theories, it should also be of interest to study the symmetries of other two dimensional string theories such as the ones compactified on manifolds other than the tori, such as $K_{3} \times T^{4}$ or a product of Calabi-Yau with $T^{2}$ and so on. Progress on these topics will hopefully be reported in near future. 


\section{References}

[1] J. Schwarz, Caltech Preprints, hepth/9503127; hepth/9505170; D. Olive, Lectures at the Spring School on String Theory, ICTP Trieste, 1995, to appear in the proceedings.

[2] E. Witten, Institute of Advanced Study Preprint, hepth/9503124.

[3] E. Witten, Institute of Advanced Study Preprint, hepth/9505186.

[4] A. Sen, Int. J. Mod. Phys.A9 (1994) 3707 and references therein.

[5] J. H. Schwarz and A. Sen Nucl. Phys. B411 (1994) 35; Phys. Lett. B312; (1993) 105.

[6] A. Sen, Nucl. Phys. B434 (1995) 179.

[7] A. Sen, Tata Institute Preprint, hepth/9503057.

[8] A. Sen, Tata Institute Preprint, hepth/9504027.

[9] N. Seiberg and E. Witten, Nucl. Phys. B426 (1994) 19; Nucl. Phys. B431 (1994) 484.

[10] C. Hull and P. Townsand, hepth/9410167.

[11] P. Breitenlohner and D. Maison, in Lecture Notes in Physics Vol. 205, Eds. C. Hoenselaers and W. Dietz, Springer-Verlag, (1984) and references therein; B. C. Xanthopoulous, in Lecture Notes in Physics Vol. 239, Ed. R. Martini, Springer-Verlag (1985) and references therein.

[12] J. Ehlers, Dissertation, Hamburg (1957); F. J. Ernst, Phys. Rev.167 (1968) 1175.

[13] R. Geroch, J. Math Phys.13 (1972) 394.

[14] W. Kinnersley, J. Math Phys.18 (1977) 1529. 
[15] W. Kinnersley and D. M. Chitre, J. Math Phys.18 (1977) 1538.

[16] W. Kinnersley and D. Chitre, J. Math Phys. 19 (1978) 1926; ibid 19 (1978) 2037.

[17] D. Cox and W. Kinersley, J. Math Phys. 20 (1979) 1225; C. Hoenselaers, W. kinnersley and B. Xanthopoulos, J. Math Phys. 20 (1979) 2530; W. Kinnersley, J. Math Phys. 21 (1980) 2231.

[18] H. Nicolai, Phys. Lett. B194 (1987) 402.

[19] B. de Wit and H. Nicolai, Nucl. Phys. B208 (1982) 323; H. Nicolai and N. P. Warner, Comm. Math. Phys.125 (1989) 369; D. Korotkin and H. Nicolai, Phys. Rev. Lett.74 (1995) 1272.

[20] I. Bakas, Nucl. Phys. B428 (1994) 374.

[21] J. H. Schwarz, Lecture in Puri Workshop on Physics at the Planck Scale, December 1994, to appear in the Proceedings.

[22] J. Maharana, Bhubaneswar Preprints IP/BBSR/95-9 (hep-th/9502001), IP/BBSR/955 (hep-th/9502002).

[23] J. Schwarz, Caltech Preprint, hepth/9503078.

[24] D. Galtsov and O. Kechkin, Phy. Rev. D50 (1994) 7394.

[25] K. Ray and A. Kumar, Bhubaneswar Preprint, hepth/9503154 (to appear in Phys. Lett. B).

[26] I. Bakas, Phys. Lett. B343 (1995) 103.

[27] A. Sen Phys. Lett. B271 (1991) 295. 
[28] A. Tomimatsu and H. Sato, Prog. Theor. Phys. 50 (1973) 95; D. Kramer, H Stephani, M. McCallum, Exact Solutions of Einstein's Field Equations, Cambridge Univ. Press, 1980.

[29] A. Biswas, K. Ray and A. Kumar, in preparation.

[30] H. Boonstra and M. de Roo, Groningen Preprint, hepth/9503038. 